\section{Possibilities and challenges for physical and social environment research in Brazil: a systematic literature review on health behaviors}

\author{
Possibilidades e desafios para pesquisas sobre \\ ambiente físico e social no Brasil: uma revisão \\ sistemática da literatura sobre comportamentos \\ de saúde
}

Posibilidades y desafíos para la investigación sobre el entorno físico y social en Brasil: una revisión sistemática de la literatura sobre conductas de salud

\begin{abstract}
This systematic review analyzed articles focused on the relationship between environment (physical, built, perceived, and social) and smoking, alcohol drinking, physical activity, diet, and obesity in Brazil. Studies published between 19952011 were retrieved from seven databases and hand searches. Based on the 42 articles reviewed, gaps were identified and recommendations were made for future research. Despite a growing number of studies, the Brazilian literature is still limited. The increase of articles in 2010-2011 coincided with the diversification of lifestyles studied, although physical activity domain remains predominant. Most studies analyzed neighborhood settings and used subjective measures for lifestyle and for environment. The presence of recreational facilities was the main physical environment aspect studied, while safety from crime was the prominent social environment factor. More research is needed to yield a rich body of evidence that leads to theoretical and methodological advances, and that supports interventions aimed at creating healthy environments.
\end{abstract}

Environment and Public Health; Health Behavior; Obesity; Review
Ana Paula Belon 1

Candace Nykiforuk 1

\section{Resumo}

Esta revisão sistemática avaliou artigos focados na relação entre ambiente (físico, construido, percebido e social) e tabagismo, consumo de álcool, prática de atividade física, hábito alimentar e obesidade no Brasil. Estudos publicados entre 1995 e 2011 foram identificados em sete bases de dados e em buscas manuais. Baseado nos 42 artigos revisados, lacunas foram identificadas e recomendações feitas para futuros estudos. Apesar da crescente produção, a literatura ainda é limitada. O maior número de artigos em 2010-2011 coincidiu com uma ligeira diversificação dos estilos de vida estudados, embora as atividades físicas ainda predominem. Muitos estudos analisaram vizinhanças e usaram medidas subjetivas para estilos de vida e fatores ambientais. A disponibilidade de espaços de recreação foi o principal fator de ambiente físico avaliado, enquanto que a questão de segurança se destacou no ambiente social. É preciso produzir mais evidências para contribuir com avanços no campo teórico e metodológico e com o delineamento de intervenções voltadas para criar espaços saudáveis.

Meio Ambiente e Saúde Pública; Comportamentos Saudáveis; Obesidade; Revisão 


\section{Introduction}

Evidence supports that the places where people live, study and work create opportunities for or barriers to engage in healthy lifestyles 1,2,3. Due to the increasing prevalence of chronic diseases worldwide and the limited effectiveness of individual-based interventions in long-term behavioral change 4,5,6, a renewed interest in the environment has drawn attention to the influences of structural and environmental conditions on health behaviors since 1990 7,8,9.

Oriented mainly by socioecological models 7 , a large number of studies in this research track have sought to understand how the features of the areas where people live might be healthdamaging or health-promoting. In other words, an individual's surrounding environment may inhibit or foster health and well-being as it provides the broader context of opportunities (e.g., supportive infrastructure for physical activity, farmer's markets to improve access to fresh fruits and vegetables, and smoke-free zones) for people to develop and maintain (un)healthy choices 10,11. From multi-level models with complex causal pathways, one understands that health outcomes would be the result of the interaction between individual characteristics (e.g., income, occupation, and education level) and environmental factors $12,13,14,15$.

The impact of physical and social environment on lifestyles has been widely investigated in high-income countries 16,17 , where the identification of the environmental determinants of health behaviors has stimulated the debate with national and local authorities about appropriate actions to support health promotion 5,18. From a solid and consistent body of evidence, this knowledge has been translated into practice, leading to the development of several initiatives, such as embellishment of existent areas to promote physical activities and school interventions to increase the consumption of vegetables and fruits 10,12,19.

The environment, or areas where people live, has been conceptualized in different ways by the research community. In general, environment can be divided into physical environment (including natural and built environment) and social environment. Natural environment refers to weather, green areas, air quality, among others. Built environment attributes, in turn, encompass neighborhood design factors, mixed land use, density, aesthetical attributes, availability of and access to facilities (e.g., parks, recreational facilities, and liquor stores). Finally, social environment features range from feelings of personal safety to policies and media promoting (un)healthy lifestyles.
To date, few studies addressing the influences of the physical and social environment on healthy lifestyles are being carried out in lowand middle-income countries. Particularly, in Brazil, despite an increasing number of studies on this topic, the evidence base in the literature is limited and provides insufficient information to support decision makers in the development and implementation of interventions aimed at creation of environments that are more conducive to healthy behaviors 20,21 .

Therefore, the aim of this review was to analyze peer-reviewed articles that have applied theoretical and/or methodological approaches related to environment (physical, built, perceived, and social) and lifestyles (tobacco use, alcohol consumption, physical activity, and eating habits) or obesity in Brazil. The literature review focuses only on these selected lifestyles and obesity since they are common risk factors for chronic diseases and, thus, important targets for prevention and control strategies. This study describes trends and patterns of the literature reviewed, as well as the conceptualization, operationalisation, and measurement of the environment. The purpose of this paper was to summarize the main characteristics of the existing body of literature, identify gaps in the knowledge and, consequently, provide recommendations for future research for environment and health in Brazil.

\section{Methods \\ Search strategy and article selection}

The systematic review examined peer-reviewed articles, including systematic literature reviews, validation studies, evaluation studies, and qualitative and quantitative studies. The articles met the following inclusion criteria: (1) analyzed any characteristic of the relationship between physical, built, perceived, or social environment and smoking, alcohol misuse, absence of physical activity, poor diet, or obesity in the Brazilian context; (2) published between January 1st, 1995 and December 31th, 2011; (3) written in Portuguese or English languages; (4) were peer-reviewed articles published in academic journals. Ulrich's International Periodicals Directory was used to exclude trade journals and popular magazines indexed by physical activity databases. Papers describing some policies and programs (i.e., without analysis of environmental or behavioral factors) or focusing on theoretical discussion only were excluded. The Brazilian literature included in this review comprised any article that addressed the topic of environment and lifestyles 
in Brazil, regardless of the affiliation of authors or the country where the journals were published. The choice for starting the search in 1995 was based on the resurgence of academic interest in the influences of the physical and social environment in health in the mid-1990s 7,8.

Using English and Portuguese search terms, peer-reviewed literature was retrieved from the following electronic databases: LILACS (1982 to the present), MEDLINE (1948 to the present, also including Medline(R) In-Process \& Other Non-Indexed Citations), PubMed (1950 to the present), SciELO Brazil (1998 to the present), Sociological Abstracts (1952 to the present), SportDiscus (1939 to 2011), and Web of Science (1990 to the present). Due to the limitations of electronic searching 22,23,24, we performed reference list searching, hand-searching relevant journals, and author-name searching in order to identify a comprehensive range of studies. Moreover, publications of key authors were searched for on the Plataforma Lattes website (http://lattes.cnpq. $\mathrm{br} /$ ), which is an open database of the National Council for Scientific and Technological Development (CNPq) containing the curriculum vitae of the Brazilian scientific community.

The following keywords and their variants related to the environment were used: built environment, physical environment, social environment, perceived environment, healthy environment, environmental perception, environment design, and environmental factors. To capture lifestyles, the search terms included, but were not limited to: lifestyle, walkability, walking, commuting, active living, sports, physical activity, leisure time, sedentary, tobacco, smoking, alcohol, obesity, body mass index, dietary, eating, and unhealthy food. In the electronic search, these keywords were searched within titles and abstracts for environment factors and for lifestyles. To restrict the search of articles to a focus on the Brazilian context, the words Brazil and Brazilian were searched in the title, abstract, authors' affiliation, and main text. The search log containing the full list of terms used for each database is available from the corresponding author upon request.

To verify if the articles retrieved met the inclusion criteria, two reviewers (the main author and a trained researcher assistant) independently screened the titles and abstracts of all articles initially identified, using a strict protocol (available upon request). In case of any disagreement between the reviewers, decisions were made through discussions among the two authors and the research assistant. When the exact focus of the article was not clear, the reviewers read the full text if it was written in English; otherwise, the main author conducted this task. RefWorks software (RefWorks-COS, Bethesda, USA) was used as reference manager. The review protocol is not registered in any protocol database.

\section{Data extraction}

Figure 1 illustrates the search flow of the literature review process. By July 2012, the literature search across the electronic databases retrieved 966 articles. After removing duplicate references $(n=343)$ and off-topic articles $(n=510), 113$ potentially eligible articles were selected to undergo full article review (inter-rater agreement = $91 \%)$. The majority of the publications excluded had used the term environment in another connotation than physical and social environment (e.g., domestic violence). The full article review resulted in a total of 42 articles deemed appropriate for analysis $16,17,20,21,25,26,27,28,29,30,31,32,33,34$, $35,36,37,38,39,40,41,42,43,44,45,46,47,48,49,50,51,52,53,54,55,56$, $57,58,59,60,61,62$.

The information from the articles selected was extracted by one researcher (the main author) and was checked by the second author for accuracy and completeness, if the full text was in English. For data extraction, we used a closeand open-ended questions form, which was developed in the Microsoft Access 2007 software (Microsoft Corp., USA). The following information was identified: citation, language, objective, study design, population, setting, measures of environment and lifestyles, main findings, recommendations related to the environment, and conclusions.

\section{Data analysis}

Basic bibliometric analysis 63 was undertaken to examine trends and patterns in the literature, which are essential to better understand the development and breadth of the physical and social environment research areas in Brazil. Frequency analyses were calculated for all coded variables. Multiple entries for some variables might be reported from one study; therefore, the sum of the categories of these variables might exceed the total number of articles included. For instance, papers whose samples' age ranges fit into multiple pre-defined categories $(<18,18-59,60$ and over $)$ were counted more than once; studies analyzing more than one lifestyle also contributed multiple records.

The identification of the levels or sizes of environment analyzed by the articles was based on the Analysis Grid for Environments Linked to Obesity (ANGELO) 15. Although this conceptual framework was originally developed to 
Figure 1

PRISMA flow diagram of included and excluded articles in the systematic review. Brazil, 1995-2011.

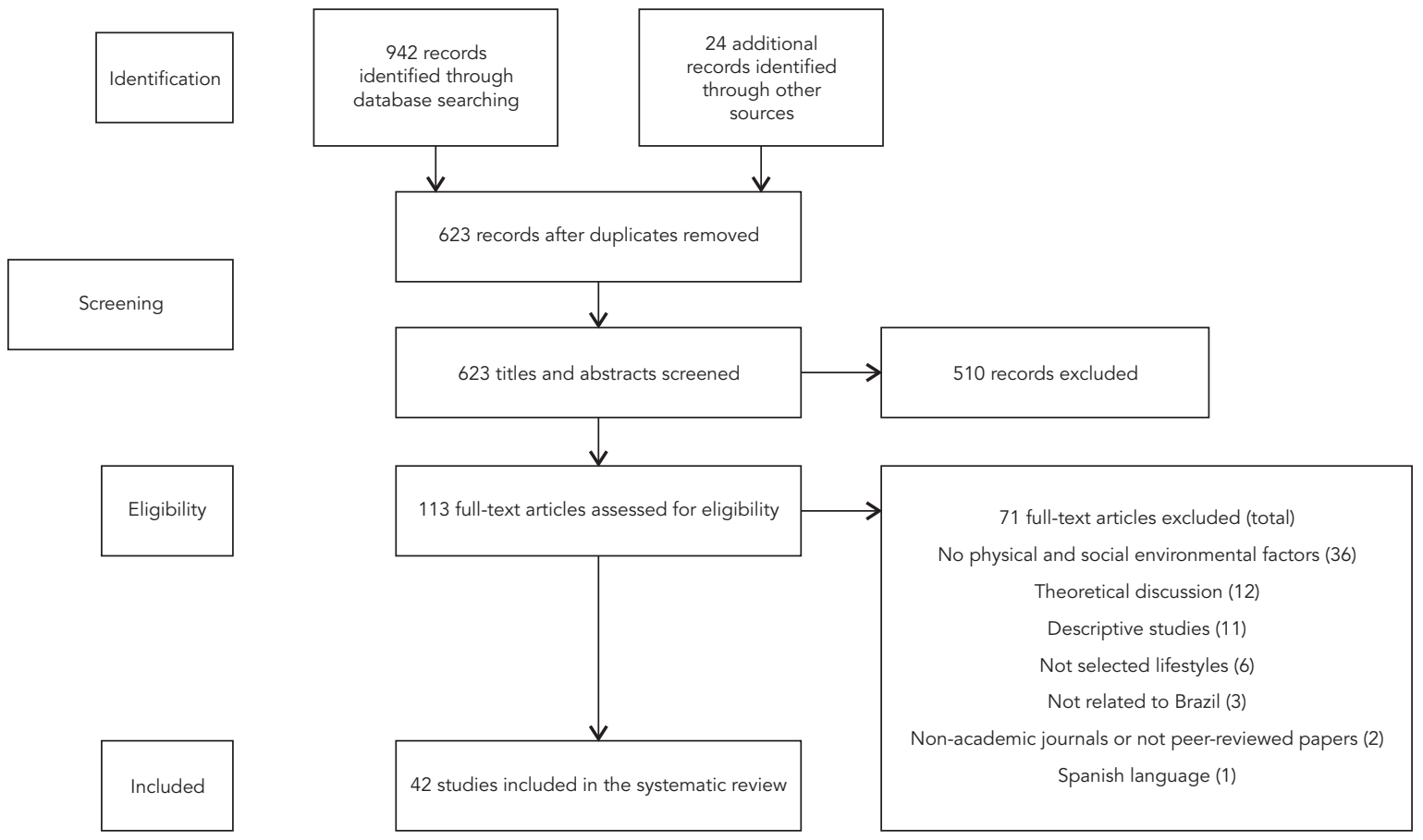

examine obesogenic environments, it has proved useful in other health settings, such as smoking and alcohol misuse 64 . The framework is a $2 \times 4$ grid that classifies the environment according to its size (micro-environmental settings and the macro-environmental sectors) and its type (physical, economic, political, and socio-cultural). Due to the small number of articles focused on Brazil, the axis related to the type of environment was not used. According to the ANGELO framework, micro-environment involves interactions between people or groups in a small area (e.g., workplaces, schools, and neighborhoods), whereas macro-environment includes broader sectors (e.g., media, government, and group of industries) that influence various settings in a diffuse way.

\section{Results}

Table 1 summarizes the main characteristics of each of the 42 articles included in this systematic review. Bibliometric characteristics and their relative frequency are shown in Table 2. Although researchers affiliated to Brazilian universities and research centers were the first authors in 33 out of 42 articles included (78.6\%), there was a strong presence of foreign researchers and professors in the authorship (42.9\%), with a predominance of those from the United States (82.4\%). Most studies were written in English, which is not surprising given the volume of papers published in nonBrazilian journals (69\%). The vast majority of articles were published in journals whose main topics were public health and safety and physical fitness and hygiene. About $65 \%$ of the papers were published between 2010 and 2011. This review also revealed a distinct concentration of papers produced by a small group of academics, who have worked mainly in the physical activity domain (data not shown).

Table 3 presents some methodological characteristics of the articles reviewed. Studying the relationship between environmental factors and lifestyles was the main objective in 40 out of 42 articles (95.2\%). Most studies used crosssectional designs. Only three qualitative articles (7.1\%) and one ecological study (2.4\%) were identified. Among the quantitative studies, the 
Articles examining the relationship between physical environment and social environment and lifestyle factors. Brazil, $1995-2011$.

\begin{tabular}{|c|c|c|c|c|c|c|c|}
\hline Reference & Year & Location & Study design & Study population & $\begin{array}{l}\text { Environment } \\
\text { assessment }\end{array}$ & $\begin{array}{l}\text { Lifestyles } \\
\text { assessment }\end{array}$ & Lifestyles studied \\
\hline $\begin{array}{l}\text { Amorim et } \\
\text { al. } 25\end{array}$ & 2010 & Pelotas/RS & $\begin{array}{l}\text { Cross- } \\
\text { sectional }\end{array}$ & $\begin{array}{l}972 \text { men and women } \\
\text { aged } 20-69 \text { years }\end{array}$ & Self-reported & Self-reported & Physical activities \\
\hline $\begin{array}{l}\text { Bandoni et } \\
\text { al. } 26\end{array}$ & 2010 & São Paulo/SP & $\begin{array}{l}\text { Cross- } \\
\text { sectional }\end{array}$ & $\begin{array}{c}1,344 \text { workers aged } \\
18 \text { years or older }\end{array}$ & Self-reported & Self-reported & Eating habits \\
\hline $\begin{array}{l}\text { Bandoni et } \\
\text { al. } 27\end{array}$ & 2011 & São Paulo/SP & Intervention & $\begin{array}{l}29 \text { companies; } 1,296 \\
\text { workers aged } 18 \text { years } \\
\text { or older }\end{array}$ & $\begin{array}{c}\text { Objective } \\
\text { (administrative } \\
\text { database) }\end{array}$ & Self-reported & Eating habits \\
\hline $\begin{array}{l}\text { Barros et } \\
\text { al. } 28\end{array}$ & 2009 & $\begin{array}{c}\text { Florianópolis/SC and } \\
\text { Recife/PE }\end{array}$ & Intervention & $\begin{array}{c}\text { 2,155 high school } \\
\text { students aged } 15-24 \\
\text { years }\end{array}$ & $\begin{array}{l}\text { Objective } \\
\text { (project's } \\
\text { activities) }\end{array}$ & Self-reported & Physical activities \\
\hline $\begin{array}{l}\text { Boscatto } \\
\text { et al. } 29\end{array}$ & 2011 & Florianópolis/SC & $\begin{array}{l}\text { Cross- } \\
\text { sectional }\end{array}$ & $\begin{array}{l}30 \text { patients who } \\
\text { underwent a bariatric } \\
\text { surgery (mean age: } \\
39.4 \pm 10.5 \text { years) }\end{array}$ & Self-reported & Self-reported & Physical activities \\
\hline $\begin{array}{l}\text { Cardoso et } \\
\text { al. } 30\end{array}$ & 2011 & Rio de Janeiro/RJ & $\begin{array}{l}\text { Cross- } \\
\text { sectional }\end{array}$ & $\begin{array}{c}1,632 \text { students in the } \\
\text { last year of primary } \\
\text { education (mean age: } \\
15.1 \text { years) }\end{array}$ & $\begin{array}{l}\text { Objective } \\
\text { (audit and } \\
\text { administrative } \\
\text { database) }\end{array}$ & $\begin{array}{l}\text { Objective (weight } \\
\text { and height were } \\
\text { measured for } \\
\text { body mass index) }\end{array}$ & Overweight \\
\hline $\begin{array}{l}\text { Cassou et } \\
\text { al. } 31\end{array}$ & 2011 & Curitiba/PR & Focus group & $\begin{array}{c}25 \text { women aged } 60 \\
\text { years or older }\end{array}$ & Self-reported & Self-reported & Physical activities \\
\hline $\begin{array}{l}\text { Chow et } \\
\text { al. } 32\end{array}$ & 2010 & $\begin{array}{c}\text { China, India, Colombia, } \\
\text { Brazil (Angatuba/SP), } \\
\text { and Canada }\end{array}$ & $\begin{array}{l}\text { Development } \\
\text { and validation } \\
\text { of the } \\
\text { instrument }\end{array}$ & $\begin{array}{l}93 \text { urban and rural } \\
\text { communities }\end{array}$ & $\begin{array}{l}\text { Self-reported } \\
\text { and objective } \\
\text { (audit) }\end{array}$ & Not applicable & $\begin{array}{l}\text { Physical activities, } \\
\text { tobacco smoking, eating } \\
\text { habits, and alcohol } \\
\text { consumption }\end{array}$ \\
\hline $\begin{array}{l}\text { Corseuil et } \\
\text { al. } 33\end{array}$ & 2011 & Florianópolis/SC & $\begin{array}{l}\text { Cross- } \\
\text { sectional }\end{array}$ & $\begin{array}{l}\text { 1,652 men and } \\
\text { women aged } 60 \text { years } \\
\text { or older }\end{array}$ & Self-reported & Self-reported & Physical activities \\
\hline $\begin{array}{l}\text { Dambros } \\
\text { et al. } 34\end{array}$ & 2011 & Santa Maria/RS & $\begin{array}{l}\text { Cross- } \\
\text { sectional }\end{array}$ & $\begin{array}{c}424 \text { high school } \\
\text { students aged 14-18 } \\
\text { years }\end{array}$ & Self-reported & Self-reported & Physical activities \\
\hline $\begin{array}{l}\text { Farias } \\
\text { Júnior et } \\
\text { al. } 35\end{array}$ & 2011 & João Pessoa/PB & $\begin{array}{l}\text { Cross- } \\
\text { sectional }\end{array}$ & $\begin{array}{l}2,874 \text { adolescents } \\
\text { aged } 14-19 \text { years }\end{array}$ & Self-reported & Self-reported & Physical activities \\
\hline $\begin{array}{l}\text { Florindo et } \\
\text { al. } 36\end{array}$ & 2009 & $\begin{array}{l}\text { State capitals and } \\
\text { Federal District }\end{array}$ & $\begin{array}{l}\text { Cross- } \\
\text { sectional }\end{array}$ & $\begin{array}{l}\text { 54,369 men and } \\
\text { women aged } 18 \text { years } \\
\text { or older }\end{array}$ & Self-reported & Self-reported & Physical activities \\
\hline $\begin{array}{l}\text { Florindo et } \\
\text { al. } 37\end{array}$ & 2011 & $\begin{array}{c}\text { Ermelino Matarazzo } \\
\text { district in São Paulo/SP }\end{array}$ & $\begin{array}{l}\text { Cross- } \\
\text { sectional }\end{array}$ & $\begin{array}{l}890 \text { men and women } \\
\text { aged } 18 \text { years or older }\end{array}$ & Self-reported & Self-reported & Physical activities \\
\hline $\begin{array}{l}\text { Gobbi et } \\
\text { al. } 38\end{array}$ & 2008 & Rio Claro/SP & $\begin{array}{l}\text { Cross- } \\
\text { sectional }\end{array}$ & $\begin{array}{c}30 \text { institutionalized } \\
\text { elderly aged } 60 \text { years } \\
\text { or older }\end{array}$ & Self-reported & Self-reported & Physical activities \\
\hline $\begin{array}{l}\text { Gomes et } \\
\text { al. } 39\end{array}$ & 2011 & $\begin{array}{c}\text { Recife/PE, Curitiba/SC, } \\
\text { and Vitória/ES }\end{array}$ & $\begin{array}{l}\text { Cross- } \\
\text { sectional }\end{array}$ & $\begin{array}{l}\text { 6,166 men and } \\
\text { women aged } 18 \text { years } \\
\text { or older }\end{array}$ & Self-reported & Self-reported & Physical activities \\
\hline $\begin{array}{l}\text { Guedes et } \\
\text { al. } 40\end{array}$ & 2011 & $\begin{array}{l}\text { Vale do Jequitinhonha/ } \\
\text { MG }\end{array}$ & $\begin{array}{l}\text { Cross- } \\
\text { sectional }\end{array}$ & $\begin{array}{c}5,100 \text { students aged } \\
6-18 \text { years }\end{array}$ & Self-reported & $\begin{array}{l}\text { Objective (weight } \\
\text { and height were } \\
\text { measured for BMI) }\end{array}$ & Overweight/Obesity \\
\hline $\begin{array}{l}\text { Hallal et } \\
\text { al. } 41\end{array}$ & 2010 & Recife/PE & $\begin{array}{l}\text { Cross- } \\
\text { sectional }\end{array}$ & $\begin{array}{l}\text { 2,046 men and } \\
\text { women aged } 16 \text { years } \\
\text { or older }\end{array}$ & Self-reported & Self-reported & Physical activities \\
\hline
\end{tabular}

(continues) 
Table 1 (continued)

\begin{tabular}{|c|c|c|c|c|c|c|c|}
\hline Reference & Year & Location & Study design & Study population & $\begin{array}{c}\text { Environment } \\
\text { assessment }\end{array}$ & $\begin{array}{c}\text { Lifestyles } \\
\text { assessment }\end{array}$ & Lifestyles studied \\
\hline $\begin{array}{l}\text { Hino et } \\
\text { al. } 43\end{array}$ & 2010 & Curitiba/PR & $\begin{array}{l}\text { Cross- } \\
\text { sectional }\end{array}$ & $\begin{array}{c}4 \text { public parks and } 4 \\
\text { squares; } 7,937 \text { public } \\
\text { park and square users } \\
\text { at all ages }\end{array}$ & $\begin{array}{l}\text { Objective } \\
\text { (audit) }\end{array}$ & Objective (audit) & Physical activities \\
\hline $\begin{array}{l}\text { Hino et } \\
\text { al. } 44\end{array}$ & 2011 & Curitiba/PR & $\begin{array}{l}\text { Cross- } \\
\text { sectional }\end{array}$ & $\begin{array}{l}\text { 1,206 men and } \\
\text { women aged } 16 \text { years } \\
\text { or older }\end{array}$ & $\begin{array}{l}\text { Objective } \\
\text { (routine } \\
\text { government } \\
\text { database) }\end{array}$ & Self-reported & Physical activities \\
\hline $\begin{array}{l}\text { Hoehner } \\
\text { et al. } 20\end{array}$ & 2008 & Brazil and Latin America & $\begin{array}{l}\text { Systematic } \\
\text { review }\end{array}$ & Not applicable & Not applicable & Not applicable & Physical activities \\
\hline $\begin{array}{l}\text { Jaime et } \\
\text { al. } 21\end{array}$ & 2011 & São Paulo/SP & $\begin{array}{l}\text { Ecological } \\
\text { study }\end{array}$ & $\begin{array}{c}31 \text { submunicipalities; } \\
2,122 \text { men and } \\
\text { women aged } 18 \text { years } \\
\text { or older }\end{array}$ & $\begin{array}{c}\text { Objective } \\
\text { (Routine } \\
\text { government } \\
\text { databases, } \\
\text { commercial } \\
\text { databases, and } \\
\text { census data) }\end{array}$ & Self-reported & $\begin{array}{c}\text { Physical activities, eating } \\
\text { habits, and overweight/ } \\
\text { obesity }\end{array}$ \\
\hline $\begin{array}{l}\text { Malavasi } \\
\text { et al. } 45\end{array}$ & 2007 & Florianópolis/SC & $\begin{array}{c}\text { Development } \\
\text { and validation } \\
\text { of the } \\
\text { instrument }\end{array}$ & $\begin{array}{l}30 \text { men and women } \\
\text { (mean age: } 27 \text { years) } \\
\text { in the } 1 \text { st phase; } 75 \\
\text { men and women } \\
\text { (mean age: } 33 \text { years) } \\
\text { in the } 2 \text { nd phase }\end{array}$ & Self-reported & Not applicable & Physical activities \\
\hline $\begin{array}{l}\text { Martins et } \\
\text { al. } 46\end{array}$ & 2000 & Not determined & $\begin{array}{c}\text { Development } \\
\text { and validation } \\
\text { of the } \\
\text { instrument }\end{array}$ & $\begin{array}{l}16 \text { men and women in } \\
\text { the } 1 \text { st phase; } 26 \text { men } \\
\text { and women in the } 2 \text { nd } \\
\text { phase }\end{array}$ & Self-reported & Not applicable & Physical activities \\
\hline $\begin{array}{l}\text { Matsudo } \\
\text { et al. } 47\end{array}$ & 2002 & São Paulo/SP & $\begin{array}{l}\text { Cross- } \\
\text { sectional }\end{array}$ & 645 households & Self-reported & Self-reported & Physical activities \\
\hline $\begin{array}{l}\text { Matsudo } \\
\text { et al. } 48\end{array}$ & 2006 & State of São Paulo & $\begin{array}{l}\text { Evaluation } \\
\text { study }\end{array}$ & Not applicable & Not applicable & Not applicable & Physical activities \\
\hline $\begin{array}{l}\text { Mendonça } \\
\text { et al. } 49\end{array}$ & 2010 & Aracaju/SE & $\begin{array}{l}\text { Cross- } \\
\text { sectional }\end{array}$ & $\begin{array}{l}\text { 2,267 men and } \\
\text { women aged } 20 \text { years } \\
\text { or older }\end{array}$ & Self-reported & Self-reported & Physical activities \\
\hline $\begin{array}{l}\text { Parra et } \\
\text { al. } 50\end{array}$ & 2010 & Recife/PE & $\begin{array}{l}\text { Cross- } \\
\text { sectional }\end{array}$ & $\begin{array}{c}10 \text { parks sites; } 32,974 \\
\text { public park users at } \\
\text { all ages }\end{array}$ & $\begin{array}{l}\text { Objective } \\
\text { (audit) }\end{array}$ & Objective (audit) & Physical activities \\
\hline $\begin{array}{l}\text { Parra et } \\
\text { al. } 16\end{array}$ & 2011 & Curitiba/PR & $\begin{array}{l}\text { Cross- } \\
\text { sectional }\end{array}$ & $\begin{array}{l}\text { 2,008 men and } \\
\text { women aged } 18 \text { years } \\
\text { or older }\end{array}$ & Self-reported & Self-reported & Physical activities \\
\hline $\begin{array}{l}\text { Petroski et } \\
\text { al. } 51\end{array}$ & 2009 & Curitiba/PR & $\begin{array}{l}\text { Cross- } \\
\text { sectional }\end{array}$ & $\begin{array}{l}218 \text { public park users } \\
\text { aged } 18 \text { years or older }\end{array}$ & Self-reported & Self-reported & Physical activities \\
\hline $\begin{array}{l}\text { Reis et } \\
\text { al. } 52\end{array}$ & 2009 & Curitiba/PR & $\begin{array}{l}\text { Cross- } \\
\text { sectional }\end{array}$ & $\begin{array}{c}\text { 1,650 high school } \\
\text { students aged } 14-18 \\
\text { years }\end{array}$ & Self-reported & Self-reported & Physical activities \\
\hline $\begin{array}{l}\text { Reis et } \\
\text { al. } 53\end{array}$ & 2010 & Curitiba/PR & $\begin{array}{l}\text { Cross- } \\
\text { sectional }\end{array}$ & $\begin{array}{l}\text { 2,097 men and } \\
\text { women aged } 18 \text { years } \\
\text { or older }\end{array}$ & Self-reported & Self-reported & Physical activities \\
\hline
\end{tabular}

(continues) 


\begin{tabular}{|c|c|c|c|c|c|c|c|}
\hline Reference & Year & Location & Study design & Study population & $\begin{array}{l}\text { Environment } \\
\text { assessment }\end{array}$ & $\begin{array}{l}\text { Lifestyles } \\
\text { assessment }\end{array}$ & Lifestyles studied \\
\hline $\begin{array}{l}\text { Sallis et } \\
\text { al. } 54\end{array}$ & 2009 & $\begin{array}{l}\text { Belgium, Brazil, Canada, } \\
\text { Colombia, China (Hong } \\
\text { Kong), Japan, Lithuania, } \\
\text { New Zealnad, Norway, } \\
\text { Sweden, and United } \\
\text { States }\end{array}$ & $\begin{array}{l}\text { Cross- } \\
\text { sectional }\end{array}$ & $\begin{array}{l}11541 \text { individuals } \\
\text { aged } 18-65 \text { years }\end{array}$ & Self-reported & Self-reported & Physical activities \\
\hline $\begin{array}{l}\text { Salvador } \\
\text { et al. } 17\end{array}$ & 2009 & $\begin{array}{l}\text { Ermelino Matarazzo } \\
\text { district in São Paulo/SP }\end{array}$ & $\begin{array}{l}\text { Cross- } \\
\text { sectional }\end{array}$ & $\begin{array}{l}385 \text { men and women } \\
\text { aged } 60 \text { or older }\end{array}$ & Self-reported & Self-reported & Physical activities \\
\hline $\begin{array}{l}\text { Salvador } \\
\text { et al. } 55\end{array}$ & 2010 & $\begin{array}{l}\text { Ermelino Matarazzo } \\
\text { district in São Paulo/SP }\end{array}$ & $\begin{array}{l}\text { Cross- } \\
\text { sectional }\end{array}$ & $\begin{array}{l}385 \text { men and women } \\
\text { aged } 60 \text { years or older }\end{array}$ & Self-reported & Self-reported & Physical activities \\
\hline $\begin{array}{l}\text { Santos et } \\
\text { al. } 56\end{array}$ & 2010 & Curitiba/PR & Focus group & $\begin{array}{c}59 \text { high school } \\
\text { students aged 15-18 } \\
\text { years }\end{array}$ & Self-reported & Self-reported & Physical activities \\
\hline $\begin{array}{l}\text { Santos et } \\
\text { al. } 57\end{array}$ & 2010 & Curitiba/PR & $\begin{array}{l}\text { Cross- } \\
\text { sectional }\end{array}$ & $\begin{array}{c}1,615 \text { high school } \\
\text { students aged } 14-18 \\
\text { years }\end{array}$ & Self-reported & Self-reported & Physical activities \\
\hline $\begin{array}{l}\text { Scarinci et } \\
\text { al. } 58\end{array}$ & 2007 & Curitiba/PR & Focus group & $\begin{array}{l}108 \text { women workers } \\
\text { aged } 18 \text { years or older }\end{array}$ & Self-reported & Self-reported & Tobacco smoking \\
\hline $\begin{array}{l}\text { Silva et } \\
\text { al. } 59\end{array}$ & 2009 & Curitiba/PR & $\begin{array}{l}\text { Cross- } \\
\text { sectional }\end{array}$ & $\begin{array}{l}220 \text { public park users } \\
\text { aged } 18 \text { years or older }\end{array}$ & Self-reported & Not assessed & Physical activities \\
\hline $\begin{array}{l}\text { Silva et } \\
\text { al. } 60\end{array}$ & 2011 & Caxias do Sul/RS & $\begin{array}{c}\text { Cross- } \\
\text { sectional }\end{array}$ & $\begin{array}{c}1,622 \text { middle and high } \\
\text { school students aged } \\
11-17 \text { years }\end{array}$ & Self-reported & Self-reported & Physical activities \\
\hline $\begin{array}{l}\text { Silva et } \\
\text { al. } 61\end{array}$ & 2011 & $\begin{array}{c}\text { State of Rio Grande } \\
\text { do Sul }\end{array}$ & $\begin{array}{c}\text { Cross- } \\
\text { sectional }\end{array}$ & $\begin{array}{l}2,265 \text { industrial } \\
\text { workers aged } 18 \text { years } \\
\text { or older }\end{array}$ & Self-reported & Self-reported & Physical activities \\
\hline $\begin{array}{l}\text { Simões et } \\
\text { al. } 62\end{array}$ & 2009 & Recife/PE & $\begin{array}{l}\text { Cross- } \\
\text { sectional }\end{array}$ & $\begin{array}{c}2,038 \text { men and } \\
\text { women aged } 16 \text { years } \\
\text { or older }\end{array}$ & Self-reported & Self-reported & Physical activities \\
\hline
\end{tabular}

Brazilian states: ES: Espírito Santo; MG: Minas Gerais; PB: Paraíba; PE: Pernambuco; PR: Paraná; RJ: Rio de Janeiro; RS: Rio Grande do Sul; SC: Santa Catarina; SE: Sergipe; SP: São Paulo.

sample size ranged from 30 to 54,369. Adults and elderly people were studied almost equally; children aged less than 18 years were under-studied. Most research was carried out in only three cities: Curitiba, São Paulo, and Recife. The physical activity domain outweighed the other lifestyles investigated, whereas smoking and alcohol consumption were less studied. Subjective measures of either lifestyle (e.g., practice of physical activity and consumption of fruits and vegetables per week) or environment attributes (e.g., perceived walking distance from home to commercial establishments and perception of safety) were used in the majority of the articles. Surveys were the most common instruments used to gather information about lifestyles and environmental factors. Only two articles applied GIS (Geographic Information System) techniques to geocode environmental attributes in the neighborhood (data not shown).

To collect data on lifestyle factors, the International Physical Activity Questionnaire (IPAQ) long version 65 was the most frequently applied questionnaire. To assess the environment, Neighborhood Environmental Walkability Scale (NEWS) 66 was extensively used (Table 3). A large proportion of the articles (59.5\%) focused on micro-environment, particularly neighborhood settings. Remaining articles were distributed across recreational facilities/open spaces/ parks, workplace, and school. In $28.6 \%$ of the articles, the exact setting could not be identified. In comparison to the micro-environment, the macro-environment domain featured less prominently. Since some of the articles evaluated more than one type of macro-environment, multiple 
Bibliometric characteristics of the 42 articles included in the systematic review. Brazil, 1995-2011.

\begin{tabular}{ll}
\hline Bibliometric characteristic & \% of studies \\
\hline First author's affiliation & 78.6 \\
$\quad$ Brazilian institution & 21.4 \\
$\quad$ Non-Brazilian institution & \\
International partnership (in research team) & 42.9 \\
Yes & 57.1 \\
No & \\
Language the paper was written in & 66.7 \\
English & 21.4 \\
Portuguese & 7.1 \\
English and Portuguese & 4.8 \\
English, Portuguese, and Spanish & \\
Journal's country of origin & 31.0 \\
Brazil & 69.0 \\
Other & \\
Journal research area * & 23.8 \\
Public Health and Safety & 21.4 \\
Physical Fitness and Hygiene & 4.8 \\
Medical Sciences & 2.4 \\
Social Sciences & 2.4 \\
Psychology & 2.4 \\
Sports and Games & 42.8 \\
Interdisciplinary ** & \\
Year of publication & 11.9 \\
2000-2007 & 23.8 \\
2008-2009 & \\
2010-2011 & \\
\hline Subjetclass & \\
\hline
\end{tabular}

* Subject classification based on Ulrich's International Periodicals Directory;

** Interdisciplinary encompasses: 6 articles in Medical Sciences/Public Health and Safety; 2 in Nutrition and Dietetics/ Psychology/Physical Fitness and Hygiene; 1 in Nutrition and Dietetics/Home Economics; 3 in Nutrition and Dietetics/ Public Health and Safety; 6 in Sports and Games/Physical Fitness and Hygiene.

answers to this question were considered. Within this domain, mass media represented the factor that was analyzed most often (44.5\%). Among the articles related to public policies and programs, community-based interventions aimed at promoting physical activity in some Brazilian State capitals were the most studied, especially those analyzing Academia da Cidade ("City Gyms") in the city of Recife, in Northeastern Brazil.

Additional investigation (data not shown) indicated that few articles studied socialinequalities related to physical and social environment: only eight articles addressed the relationship between socioeconomic indicators and environment and 14 articles analyzed environmental attributes according to gender. Moreover, the review found that nine studies applied international question- naires for environment, which had not been validated in Brazil. The questionnaires were: Physical Activity Neighborhood Environment Scale (PANES) 67, NEWS-Abbreviated (NEWS-A) 68, and modified version of NEWS, whose full version had been validated in Brazil 45 .

Table 4 shows which components of physical and social environment were investigated in Brazil. Recreational facilities (71.4\%), walking and cycling infrastructure (57.1\%), and accessibility to non-residential areas $(54.8 \%)$ were the main physical environment factors studied. Safety from crime was the prominent social environment factor, accounting for $64.3 \%$ of all studies.

Further analysis (data not shown) identified that few articles used the following terms built (13), physical (10), social (10), and perceived (15) 
Methodological characteristics of the 42 articles included in the systematic review *. Brazil, 1995-2011.

\begin{tabular}{|c|c|}
\hline Characteristics & $\%$ \\
\hline \multicolumn{2}{|c|}{ One of the objectives was the relationship between environment and lifestyle ( $n=42)$} \\
\hline Yes & 95.2 \\
\hline No & 4.8 \\
\hline \multicolumn{2}{|l|}{ Study design $(n=42)$} \\
\hline Cross-sectional & 73.8 \\
\hline Experimental & 4.8 \\
\hline Qualitative studies & 7.1 \\
\hline Validation studies & 7.1 \\
\hline Systematic Review & 2.4 \\
\hline Evaluation study & 2.4 \\
\hline Ecological study & 2.4 \\
\hline \multicolumn{2}{|l|}{ Sample age (years) ${ }^{* \star}(n=80)$} \\
\hline Child/Adolescents $(<18)$ & 21.3 \\
\hline Adults (18-59) & 37.5 \\
\hline Elderly $(60+)$ & 35.0 \\
\hline Not determined & 3.8 \\
\hline Not applicable & 2.4 \\
\hline \multicolumn{2}{|l|}{ Geographic region $(s) * \star(n=45)$} \\
\hline Curitiba & 26.7 \\
\hline São Paulo & 15.7 \\
\hline Recife & 13.3 \\
\hline Florianópolis & 8.9 \\
\hline Brazil and Latin America & 2.2 \\
\hline São Paulo State & 2.2 \\
\hline Rio Grande do Sul State & 2.2 \\
\hline State capitals and Federal District & 2.2 \\
\hline Other & 22.2 \\
\hline Not determined & 4.4 \\
\hline \multicolumn{2}{|l|}{ Lifestyle factor(s) assessed $* *(n=47)$} \\
\hline Physical activity & 78.7 \\
\hline Eating habits & 8.5 \\
\hline Overweight/Obesity & 6.4 \\
\hline Smoking & 4.3 \\
\hline Alcohol consumption & 2.1 \\
\hline \multicolumn{2}{|l|}{ Lifestyle measures used $(n=42)$} \\
\hline Subjective & 76.2 \\
\hline Objective & 9.5 \\
\hline Not assessed & 2.4 \\
\hline Not applicable & 11.9 \\
\hline \multicolumn{2}{|l|}{ Environmental measures used $(n=42)$} \\
\hline Subjective & 76.2 \\
\hline Objective & 16.6 \\
\hline Both & 2.4 \\
\hline Not applicable & 4.8 \\
\hline
\end{tabular}

(continues) 


\begin{tabular}{|c|c|}
\hline Characteristics & $\%$ \\
\hline \multicolumn{2}{|l|}{ Lifestyle factor(s) data source $(n=42)$} \\
\hline Survey & 69.0 \\
\hline Focus groups & 7.1 \\
\hline Audit & 4.8 \\
\hline Measurement of body mass index & 4.8 \\
\hline Not applicable & 14.3 \\
\hline \multicolumn{2}{|c|}{ Environmental (physical or social) data source ${ }^{\star \star}(n=45)$} \\
\hline Survey & 66.7 \\
\hline Administrative database/Census & 11.0 \\
\hline Audit & 8.9 \\
\hline Focus groups & 6.7 \\
\hline Not applicable & 6.7 \\
\hline \multicolumn{2}{|c|}{ Instrument(s) used to assess lifestyle factors ** $(n=45)$} \\
\hline IPAQ (long version) & 28.9 \\
\hline Developed by the authors & 24.4 \\
\hline VIGITEL & 6.7 \\
\hline SOPARC & 4.4 \\
\hline Qualitative instruments & 6.7 \\
\hline Diary & 2.2 \\
\hline Other & 15.6 \\
\hline Not applicable & 11.1 \\
\hline \multicolumn{2}{|c|}{ Instrument(s) used to assess environment ${ }^{\star \star}(n=44)$} \\
\hline Developed by the authors & 31.8 \\
\hline NEWS/A-News & 20.5 \\
\hline Administrative database/Census & 9.1 \\
\hline SOPARC & 4.5 \\
\hline Qualitative instruments & 6.8 \\
\hline IPAQ Environmental-Module & 2.3 \\
\hline VIGITEL & 2.3 \\
\hline Other & 15.9 \\
\hline Not applicable & 6.8 \\
\hline \multicolumn{2}{|l|}{ Level of environment assessed ${ }^{\star \star \star}(n=42)$} \\
\hline Microenvironment & 59.5 \\
\hline Macroenvironment & 2.4 \\
\hline Both & 9.5 \\
\hline Not determined & 28.6 \\
\hline \multicolumn{2}{|l|}{ Micro-environment setting $(n=29)$} \\
\hline Neighborhood/Community settings & 62.1 \\
\hline Recreational facilities/Open spaces/Parks & 20.7 \\
\hline Workplace & 10.3 \\
\hline School & 6.9 \\
\hline \multicolumn{2}{|l|}{ Macro-environment setting ** $(n=9)$} \\
\hline Mass media & 44.5 \\
\hline Health promotion policies and campaigns & 33.3 \\
\hline Urban planning & 22.2 \\
\hline
\end{tabular}

(continues) 
Table 3 (continued)

\begin{tabular}{lc}
\hline Characteristics & $\%$ \\
\hline Related to public policies/programs ** $(n=15)$ & \\
Academia da Cidade (City Gyms) in Recife & 33.3 \\
Worker's food program/National School Food Program & 20.0 \\
CuritibAtiva in Curitiba & 13.3 \\
Agita São Paulo (Move for Health, São Paulo city) & 13.3 \\
Academia da Cidade (City Gyms) in Aracaju & 6.7 \\
Physical Activity Orientation Service in Vitória & 6.7 \\
Set of health promotion policies and campaigns & 6.7 \\
\hline
\end{tabular}

A-NEWS: Neighborhood Environment Walkability Survey - Abbreviated; IPAQ: International Physical Activity Questionnaires; NEWS: Neighborhood Environment Walkability Survey; SOPARC: System for Observing Play and Recreation in Communities; VIGITEL: Telephone-based Surveillance of Risk and Protective Factors for Chronic Diseases.

* Number of records in parenthesis;

** Variables with multiple answers;

*** Micro-environment and macro-environment refer to the size of environment studied, based on the ANGELO framework.

Studies on physical activity programs that did not investigate a singular micro-environment (e.g., neighborhood) were

classified as macro-environment.

Table 4

Summary of the attributes of the physical environment and social environment used by the 42 articles included in the systematic review. Brazil, $1995-2011$.

\section{Attribute by type of environment}

Physical environment

Presence of recreational facilities (e.g., gyms, parks, and soccer fields)

Presence/degree of walking and cycling infrastructure (e.g., sidewalks and bike lanes)

Accessibility or proximity to non-residential areas (e.g., recreation facilities are close to home)

Safety from traffic (e.g., drivers' respect to pedestrians)

Bad weather (e.g., too cold or windy)

Aesthetics (e.g., open-air sewage, and garbage and debris on the streets)

Mixed land use (e.g., commercial and residential in one area)

Presence/degree of street lighting

Density (e.g., number of fast-food restaurants per 1,000 inhabitants)

Degree of air or noise pollution

Steepness of streets (e.g., mean of slope)

Presence/degree of green areas

Convenience of public transport (e.g., daily frequency of buses)

Access to and availability of fruits and vegetables (e.g., in the workplace)

Distance to the stores selling alcohol

Street connectivity

Promotion of physical activity events according to the season

Other (e.g., recreation facilities are well equipped and emergency health services are available in the parks)

Social environment

Safety from crime (e.g., safe to walk during the day and at night)

Incentive policies (e.g., smoking restrictions in the workplace)

Having a companion (e.g., to exercise with)

Social support (e.g., people tease them when they are exercising)

Lack of money (e.g., to undertake healthy lifestyle behaviors)

Knowledge/participation (e.g., in the community-based physical activity programs)

Walking the dog

Partnerships (e.g., with non-governmental organizations to promote physical activity events)

Other (e.g., satisfaction with the physical activity program available in the neighborhood)
$\%$ of studies

71.4

57.1

54.8

42.9

40.5

38.1

31.0

26.2

23.8

23.8

21.4

16.7

14.3

9.5

9.5

7.1

4.8

23.8

64.3

38.1

40.5

26.2

19.0

16.7

9.5

7.1

16.7 
environment. Among them, very few articles provided direct or indirect concepts (using citations) for built (9), physical (4), social (5), and perceived (9) environment.

\section{Discussion}

The present research sought to shed light on the types of studies that have focused on the relationship between physical and social environment and health in Brazil. By describing the trends and patterns of the Brazilian literature focused on the relationship between the environment and lifestyles, this study identified gaps in the scientific production of knowledge. Based on the unaddressed topics, recommendations for future progress are provided below.

Our literature review revealed that only 42 articles were published in peer-reviewed journals between 1995 and 2011. Despite this limited amount of studies, there is a growing Brazilian literature addressing issues related to the impact of physical and social environment on lifestyles. The notable publication peak in 2010 and 2011 is indicative of the greater academic interest in this research field in Brazil. The study findings also suggested that international partnerships have boosted scientific production with the participation of co-authors from other countries in half of the articles included in this review. Particularly, the partnership with institutions from the United States in the development of studies has led to a rich and fruitful exchange of expertise in this research field, which has been converted into a rapidly growing body of publications concerning physical and social environment and health in Brazil.

Despite this recent surge in publications, one chief explanation for the small number of articles since 1995 is that few academics in Brazil are working in this research track. Many of these researchers represented in this review have expertise in physical activity domain; as a result, the growing Brazilian body of research in physical and social environment has mainly focused on this topic, which represented almost $80 \%$ of all studies included here. Physical activity has also been the main lifestyle investigated in high-income countries ${ }^{4}$. Little is known about the roles that physical and social environments play in influencing eating habits and overweight/obesity in Brazil; additionally, analysis is almost non-existent for tobacco use and alcohol consumption. There is supportive evidence from studies conducted in high-income countries that environment influences dietary behaviors 69 , overweight/obesity 4,70 , alcohol use 71 , and smoking 2 in posi- tive and negative ways. Diversifying the topics studied is, therefore, crucial to gain a better understanding of the complexity and multifaceted nature of the physical and social environment and its effects in stimulating or discouraging the engagement in healthy lifestyles. A comprehensive picture of these relationships may provide guidance to develop a national or local action plan focused on the promotion of the environments that support healthy choices. In addition, because of different environmental mechanisms affecting each particular behavior 5,14,72, the incorporation of these important and overlooked lifestyles into the research agenda may uncover which environmental features are most influential (and highest priority for intervention) in a comprehensive health-promotion agenda.

Given that only three cities (Curitiba, São Paulo, and Recife) accounted for more than half of the geographic regions examined, additional research on how environment affects the adoption of healthy lifestyles in other cities is strongly encouraged. Study replication in different settings may improve the understanding of which aspects of the physical and social environment can be health-promoting and health-damaging.

Adults and elderly people in Brazil were studied in almost similar proportion; less attention has been paid to children and adolescents. More needs to be done for such groups, since most research carried out in high-income countries has highlighted, for example, the negative impact of obesogenic environment 70,73 and of great availability of cigarettes in the school neighborhoods 2 on the health of young people. Additionally, investigating different age groups individually or comparatively is important for understanding how and to what extent the physical and social environment shapes health-related lifestyles across the lifespan 4,10 .

Our findings also showed that the current evidence for Brazil is mostly derived from crosssectional studies. The relative shortage of experimental, quasi-experimental, and longitudinal research on environment and lifestyles has also been identified in reviews conducted in highincome countries 4,13,72,73. Given their ability in determining the directionality in the association between environmental factors and lifestyles, more research should be conducted with these strong study designs to provide support to the findings from cross-sectional studies 19.

Qualitative evidence can also shed light into the relationships between environment and lifestyles, by uncovering mediating pathways and factors specific to the Brazilian context. The potential of qualitative techniques in studies on physical and social environment has been dem- 
onstrated in several articles, through which issues that are different to those found in surveys have emerged $5,18,74,75$. The only three qualitative studies included in this review confirm these strengths $31,56,58$. For instance, conducting focus groups among women in workplaces, Scarinci et al. 58 found that counter-advertising was considered a contributor to the smoking initiation by the current smokers. Such contextual information, which is barely captured through quantitative studies, may be helpful for intervention development, especially concerning smoking cessation campaigns.

With regard to the procedures for collecting data on environment, surveys were predominant; nevertheless, it is worth highlighting the use of the systematic observation by some studies. The audit tool has been considered a valuable strategy because its measurements are not affected by memory bias and are standardized, thus are more accurate in comparison to subjective measurements. Another advantage is that the systematic observation takes into account the presence and the quality of environmental attributes 76. An important limitation, however, is that systematic observation does not capture the perception of residents 9 . It is well recognized that people create different representations of the place they are in, observing and interpreting the features of their surroundings according to their socioeconomic and cultural backgrounds 3; therefore, the inclusion of these aspects related to perceptions can provide important insights on the interactions of people with the environment and guide environmental interventions that enlarge people's choices in a meaningful way to them.

Other studies have taken advantage of the availability of administrative databases on urban planning. Despite the limitations related to the temporal disconnection between health outcome and environmental attributes, the low or unknown quality of data, the lack of comparable boundaries, and exclusion of qualitative aspects of the environment 3,72 , routine statistics allow researchers, in a feasible way, to obtain objective data on a large geographic scale 76 .

Only two papers characterized the environment with GIS-based measures using existent archival data. In high-income countries, geoprocessing techniques have been increasingly incorporated into studies on environmental correlates because of their ability to produce more sophisticated analysis 5,69,76.

A large majority of articles used self-reported measures for environment and for lifestyles. Other reviews have also found that perceived environmental attributes were more frequently used than objective measures 12,73,76. Concerning lifestyles, a similar trend was seen in a review on physical activity 13,73 , whereas, for dietary behaviors, a recent review article showed that GISbased measures were the most common measures of the food environment ${ }^{69}$. Given the divergence of results between subjective and objective measures found in recent literature reviews investigating different environmental attributes and lifestyles 12,73 , the inclusion of both indicators has been strongly recommended 1,12 since this combination would aid to yield consistent data and improve understanding on interactions of people with the tangible environmental structure 5. In Brazil, particular attention should be placed on obtaining objective measures of physical and social environments since few studies are exploring this methodology. Additional steps should also be taken towards the simultaneous use of subjective and objective indicators.

Some studies used international instruments for measurement of environment that had not been validated in Brazil. For instance, based on the validated full version of NEWS in Brazil 45, some authors applied a modified short version of this questionnaire 17,33, whose validation was only carried out later by Florindo et al. 77 . Since the instrument should be culturally significant and suitable for the Brazilian context in order to yield reliable, consistent findings, the validation of international questionnaires is particularly critical in the assessment of the links between lifestyles and environment in different settings.

Concerning micro- and macro-environment, other reviews have also showed that the former is the most studied 12,70. Neighborhoods and community settings were predominant among Brazilian studies and few studies focused on macroenvironment sectors, such as mass media, health campaigns and policies, and town planning. Therefore, despite the complexity in analyzing the diffuse and interconnected effects of the macro-environment 15 , more attention should be drawn to this level. Regulations, laws, policies, institutional rules, and marketing strategies 15 exert a powerful influence in shaping food choices (including alcoholic beverage consumption) and attitudes and beliefs concerning smoking as well as engagement in active transport and recreational activities.

The small number of studies investigating the relationship between socioeconomic inequalities and physical and social environment in this review is of concern, since several studies conducted in high-income countries have pointed out that people living in deprived areas face more obstacles to make healthy choices 3,4,7,19,64. Failure to acknowledge and measure the social 
inequalities related to the environment can certainly hamper the full understanding of the relationship between environmental factors and health in a middle-income country, like Brazil.

The inclusion of socioeconomic aspects in the analysis of the impact of the environment on health has been done in two ways. The first is the investigation of how people with different socioeconomic status, for instance, perceive the environmental attributes in their neighborhood. An example of this analysis is the study of Cassou et al. 31 that, through focus groups, found that lowsocioeconomic status women perceived environmental factors as a barrier to physical activity more frequently than their high-socioeconomic status counterparts in Brazil. This data suggest that an increase in the availability of free or lowcost facilities, as well as investment in safety issues in public spaces might encourage poor people to be more physically active.

Another possibility has been to determine the geographic distribution of environmental attributes and analyze its relationship with health behaviors in light of social inequalities. A combination of (1) aggregate measures of the socioeconomic characteristics of people living in different areas (area measures related to the social context and deprivation), (2) spatial distribution of environmental attributes, and (3) individual-level health outcomes have been used by researchers in this field. The study of Jaime et al. 21, included in this review, illustrates this application. Through an ecological analysis of the relationship between environmental factors and lifestyles in São Paulo city, the authors found higher densities of food stores, public transport links, parks, and public recreational facilities in affluent areas, where overweight rates were lower. Such findings, which corroborate prior knowledge 3,70 , reinforce the need for health promotion policies targeted at improving the physical and social environment in deprived areas, providing amenities and opportunities at affordable or no cost for the local population engaging in healthy lifestyles.

One third of the articles investigated gender differences in perceptions of the environment. Given the convincing evidence about the different patterns of the relationships between environment and lifestyles according to gender $11,72,78$, there is no doubt that studying how women and men perceive and interact with their environment is important to the development of appropriate policies and programs to support health. For example, an article included in this review reported distinct associated environmental variables to the leisure-time physical activity for elderly men and women 17 .
Missing from the Brazilian literature is the use of the key terms of physical and social environment-related approaches. Although all articles reviewed are clearly linked to this topic, few have used the terms: built, physical, social or perceived environment. Instead, authors have preferred to use generic terms, such as environment, environmental conditions, community environment, structural factors, and so on. One reasonable explanation for this is that the Brazilian scientific production on physical and social environment is still at a relatively early stage; as a result, many authors are only exploring some of the environmental correlates but are not identifying their work with other research affiliated to that research field.

Among the studies that used at least one of these terms, only half of them provided their own definition of the environment or cited other references to contextualize it. Indeed, few studies provided a clear conceptualization of how the selected environmental attributes may affect health behaviors. Another issue identified was a large degree of variability in the operationalisation of environmental factors. The lack of clarity about definitions of the environment and the variability in the measurements can be related to the absence of a well-articulated conceptual rationale. Therefore, the incorporation of theoretical perspectives should be considered as a top priority in the research agenda, since clear definitions are an essential component of a plausible conceptual framework or theoretical model that would guide the selection of the environmental measures, data collection, and the identification of important environmental influences for each health domain and population group 72 .

Eighteen variables related to the physical environment were studied in the articles included in this review. The most often studied variable was the presence of recreational facilities. There is no doubt that the creation or revitalization of existent areas that are tailored to local needs, followed by the offer of specific programs in order to maximize the potential of this infrastructure, may enable physical activity 10,19 . Understudied environmental factors should be incorporated into future studies to evaluate the presence and direction of association with lifestyles in the Brazilian context, such as density of different establishments in the neighborhood, air or noise pollution, access to and availability of fruits and vegetables, and so forth.

Concerning the social environment, safety from crime was the most often studied variable, which is not surprising given that this issue is clearly relevant in Brazil. Similar to studies conducted in other countries 73,79, Brazilian articles 
have demonstrated somewhat mixed results concerning the association between safety from crime and healthy behaviors. More investigations should be carried out in Brazil, considering both objective (e.g., presence of security guards, crime and homicide rates) and subjective measures (e.g., feelings of safety and vulnerability) in order to advance the methodological discussion and to verify to what extent individual perceptions can be associated with tangible aspects of the environment. Such knowledge may provide cues for the design of effective interventions aimed mainly at safe places where instead people feel unsafe and are unlikely to engage in physical activity. However, the need for more research should not delay policy development. Based on the convincing evidence in the international literature, governments at all levels should urgently take action in enhancing safety in public spaces, through strategies such as street lighting, policing and surveillance by public or private security guards, and maintenance of public amenities ${ }^{1}$. Reducing fear of crime can not only foster engagement in a more active lifestyle, but also enhance a sense of belonging, improve social cohesion, and promote personal well-being 80 .

Some aspects of the social environment have also been less explored, such as partnerships. Available evidence suggests that partnerships with sectors other than health play an essential role in funding collaboration; besides, it is a good strategy to conciliate political will with financial resources and experience 10,19.

Some limitations should be considered when examining our findings. Since some articles might not have reported all variables used in the research, it is possible some information has not been included in this literature review. However, given that many studies modified international instruments or developed their own questionnaires, this strategy was necessary in order to prevent the introduction of an information bias when well-known questionnaires were applied. Further, the lack of information provided about methodology, mainly in articles published in international journals, created some difficulties for compiling a more detailed description of methods employed in this field. Future studies should specify the methodological procedures regarding the technical aspects of the environmental attributes. With more detailed information, a comprehensive quantitative synthesis of findings through a meta-analysis could be performed and more replicated studies could be conducted for novice researchers in this area.

It must be also noted that this review was limited to the examination of the environment relative to lifestyle behaviors and obesity; there- fore, further research should be expanded to include other health outcomes, such as indicators of health-related quality of life. It is worth mentioning that the current literature review identified a few studies investigating the influence of perceived environment on self-rated health and functional performance. In the international context, these well-studied research areas have added strong evidence to the literature by showing the influence of environment on physical functioning and mental health 3,19 , as well as support to interventions in public health and urban planning.

This review also retrieved some articles focusing only on the description of programs aimed at changing the physical and social environment, which were not included because they did not meet our selection criteria. However, further research should synthesize the evidence available from the Ministry of Health's actions and other local initiatives led by policymakers in order to publicize ongoing programs countrywide and contribute to the information exchange about overcoming obstacles, successful strategies for the engagement of communities, development of partnerships, and so on. In addition, assessing the performance of these initiatives can better guide the planning of more effective strategies in the future, while also paying attention to their contextual distinctiveness.

By including only peer-reviewed articles, our findings are likely to be affected by the publication bias. In addition to unpublished theses and dissertations, we are aware that some evidence generated by political institutions is usually published in reports, conferences proceedings, and other grey literature, none of which were included in this review. Additionally, given the volume of investigations published in non-indexed scientific journals (mainly those in physical activity field), it is recommended that future research evaluates and weights the study findings regarding their individual scientific rigor.

The variety of instruments and cut-off points used to measure behavior outcomes and environmental attributes, coupled with the limited set of studies included in the present review, precluded us from performing a meta-analysis with the quantitative articles. Due to this heterogeneity, subgroup-specific summaries could also not be reported.

On the other hand, the studies reviewed were systematically identified through a comprehensive list of search terms. The search was not limited to the health related databases, but also included physical activity and social sciences search sources. In addition, our literature review involved other search strategies, such as culling 
the reference lists of studies retrieved in the electronic search.

To our knowledge, this systematic literature review represented the first effort to analyze the Brazilian scientific production related to the physical and social environment. Throughout the full scope of literature reviewed, some unaddressed issues were revealed and some recommendations were provided to fill this knowledge gap. Irrespective of the relatively early stage of the Brazilian literature on this topic and the limitations identified, its growth is promising, as is the slight diversification of the themes studied.
Further replicated studies are needed to provide a full understanding of the relationships between physical and social environment and health lifestyles in different Brazilian contexts. Moreover, careful consideration should be given to the development of theoretical models that frame and guide the planning and analysis of studies in Brazil. More evidence to inform policy and practice is fundamental to make environmental surroundings a better place to grow, study, work, and age, providing real opportunities for every person to engage in healthy lifestyles.

\section{Resumen}

Esta revisión sistemática evaluó estudios enfocados en la relación entre el entorno (físico, construido, social y percibido) y tabaquismo, consumo de alcohol, actividad física, dieta y obesidad en Brasil. Los estudios publicados entre 1995-2011 se identificaron a través de siete bases de datos y búsqueda manual. En base a 42 artículos, se identificaron deficiencias y se realizaron recomendaciones para futuros estudios. A pesar del aumento en la producción, la literatura brasileña es todavía limitada. El incremento de artículos en 2010 2011 coincidió con una diversificación de los estilos de vida analizados, aunque todavía predominan las actividades físicas. La mayoría de los estudios examinaron barrios y usaron medidas subjetivas para el estilo de vida y los factores ambientales. La disponibilidad de espacios de ocio fue el principal factor del entorno físico evaluado. La falta de seguridad se destacó en el entorno social. Se necesitan más estudios para contribuir en los avances teóricos y metodológicos y en el diseño de intervenciones dirigidas a la creación de espacios saludables.

Medio Ambiente y Salud Pública; Conductas Saludables; Obesidad; Revisión

\section{Contributors}

A. P. Belon proposed the article and performed the data collection, analysis, and writing of the manuscript. C. Nykiforuk contributed substantially to the study design, data analysis and revision of the manuscript. All authors read and approved the final manuscript.

\section{Acknowledgments}

We thank Catherine Somerville (research assistant) for her careful work with the initial screening of the articles. A. P. Belon is funded by a postdoctoral fellowship from Capes/Brazil (9139/11-7). 


\section{References}

1. Duncan MJ, Spence JC, Mummery WK. Perceived environment and physical activity: a meta-analysis of selected environmental characteristics. Int J Behav Nutr Phys Act 2005; 2:11.

2. Lovato CY, Hsu HCH, Sabiston CM, Hadd V, Nykiforuk CIJ. Tobacco point-of-purchase marketing in school neighbourhoods and school smoking prevalence: a descriptive study. Can J Public Health 2007; 98:265-70.

3. Macintyre S, Maciver S, Sooman A. Area, class and health: should we be focusing on places or people? J Soc Policy 1993; 22:213-34.

4. Papas MA, Alberg AJ, Ewing R, Helzlsouer KJ, Gary TL, Klassen AC. The built environment and obesity. Epidemiol Rev 2007; 29:129-43.

5. Sallis JF, Cervero RB, Ascher W, Henderson KA, Kraft MK, Kerr J. An ecological approach to creating active living communities. Annu Rev Public Health 2006; 27:297-322.

6. Schmidt MI, Duncan BB, Silva GA, Menezes AM, Monteiro CA, Barreto SM, et al. Chronic non-communicable diseases in Brazil: burden and current challenges. Lancet 2011; 377:1949-61.

7. Cockerham WC. Social causes of health and disease. $2^{\text {nd }}$ Ed. Cambridge: Polity Press; 2008.

8. Macintyre S, Ellaway A, Cummins S. Place effects on health: how can we conceptualise, operationalise and measure them? Soc Sci Med 2002; 55: 125-39.

9. Proietti FA, Oliveira CL, Ferreira FR, Ferreira AD, Caiaffa WT. Context unit and systematic social observation: a review of concepts and methods. Physis (Rio J.) 2008; 18:469-82.

10. Giles-Corti B. People or places: what should be the target? J Sci Med Sport 2006; 9:357-66.

11. Humpel N, Marshall AL, Leslie E, Bauman A, Owen N. Changes in neighborhood walking are related to changes in perceptions of environmental attributes. Ann Behav Med 2004; 27:60-7.

12. Brug J. Determinants of healthy eating: motivation, abilities and environmental opportunities. Fam Pract 2008; 25 Suppl 1:i50-5.

13. McCormack G, Giles-Coiti B, Lange A, Smith T, Martin K, Pikora TJ. An update of recent evidence of the relationship between objective and selfreport measures of the physical environment and physical activity behaviours. J Sci Med Sport 2004; 7:81-92.

14. Sallis JF, Owen N, Fisher EB. Ecological models of health behavior. In: Glanz K, Rimer BK, Viswanath $\mathrm{K}$, editors. Health behavior and health education: theory, research, and practice. $4^{\text {th }}$ Ed. San Francisco: Jossey-Bass; 2008. p. 465-85.

15. Swinburn B, Egger G, Raza F. Dissecting obesogenic environments: the development and application of a framework for identifying and prioritizing environmental interventions for obesity. Prev Med 1999; 29:563-70.

16. Parra DC, Hoehner CM, Hallal PC, Ribeiro IC, Reis $\mathrm{R}$, Brownson RC, et al. Perceived environmental correlates of physical activity for leisure and transportation in Curitiba, Brazil. Prev Med 2011; 52:234-8.
17. Salvador EP, Florindo AA, Reis RS, Costa EF. Perception of the environment and leisure-time physical activity in the elderly. Rev Saúde Pública 2009; 43:972-80.

18. Clark MI, Berry TR, Spence JC, Nykiforuk C, Carlson M, Blanchard C. Key stakeholder perspectives on the development of walkable neighbourhoods. Health Place 2010; 16:43-50.

19. Rydin Y, Bleahu A, Davies M, Dávila JD, Friel S, De Grandis G, et al. Shaping cities for health: complexity and the planning of urban environments in the 21st century. Lancet 2012; 379:2079-108.

20. Hoehner CM, Soares J, Perez DP, Ribeiro IC, Joshu $\mathrm{CE}$, Pratt M, et al. Physical activity interventions in Latin America: a systematic review. Am J Prev Med 2008; 34:224-33.

21. Jaime PC, Duran AC, Sarti FM, Lock K. Investigating environmental determinants of diet, physical activity, and overweight among adults in Sao Paulo, Brazil. J Urban Health 2011; 88:567-81.

22. Aveyard H. Doing a literature review in health and social care: a practical guide. 2nd Ed. Berkshire: Open University Press; 2010.

23. Waddington $\mathrm{H}$, White H, Snilstveit B, Hombrados JG, Vojtkova M, Davies P, et al. How to do a good systematic review of effects in international development: a tool kit. J Dev Effect 2012; 4:359-87.

24. Liberati A, Altman DG, Tetzlaff J, Mulrow C, Gøtzsche PC, Ioannidis JPA, et al. The PRISMA statement for reporting systematic reviews and metaanalyses of studies that evaluate health care interventions: explanation and elaboration. BMJ 2009; 339:b2700.

25. Amorim TC, Azevedo MR, Hallal PC. Physical activity levels according to physical and social environmental factors in a sample of adults living in South Brazil. J Phys Act Health 2010; 7 Suppl 2:S204-12.

26. Bandoni DH, Bombem KCM, Marchioni DML, Jaime PC. The influence of the availability of fruits and vegetables in the workplace on the consumption of workers. Nutr Food Sci 2010; 40:20-5.

27. Bandoni DH, Sarno F, Jaime PC. Impact of an intervention on the availability and consumption of fruits and vegetables in the workplace. Public Health Nutr 2011; 14:975-81.

28. Barros MV, Nahas MV, Hallal PC, Farias Júnior JC, Florindo AA, Barros SSH. Effectiveness of a schoolbased intervention on physical activity for high school students in Brazil: the Saude na Boa project. J Phys Act Health 2009; 6:163-9.

29. Boscatto EC, Duarte MFS, Gomes MA. Stages of behavior change and physical activity barriers in morbid obese subjects. Rev Bras Cineantropom Desempenho Hum 2011; 13:329-34.

30. Cardoso LO, Castro IR, Gomes FS, Leite IC. Individual and school environment factors associated with overweight in adolescents of the municipality of Rio de Janeiro, Brazil. Public Health Nutr 2011; 14:914-22.

31. Cassou ACN, Fermino R, Añez CRR, Santos MS, Domingues MR, Reis RS. Barriers to physical activity among Brazilian elderly women from different socioeconomic status: a focus-group study. J Phys Act Health 2011; 8:126-32. 
32. Chow CK, Lock K, Madhavan M, Corsi DJ, Gilmore AB, Subramanian SV, et al. Environmental Profile of a Community's Health (EPOCH): an instrument to measure environmental determinants of cardiovascular health in five countries. PLoS One 2010; 5:e14294.

33. Corseuil MW, Schneider IJC, Silva DAS, Costa FF, Silva KS, Borges LJ, et al. Perception of environmental obstacles to commuting physical activity in Brazilian elderly. Prev Med 2011; 53:289-92.

34. Dambros DD, Lopes LFD, Santos DL. Perceived barriers and physical activity in adolescent students from a Southern Brazilian city. Rev Bras Cineantropom Desempenho Hum 2011; 13:422-8.

35. Farias Júnior JC, Lopes AS, Mota J, Santos MP, Ribeiro JC, Hallal PC. Perception of the social and built environment and physical activity among Northeastern Brazil adolescents. Prev Med 2011; 52:114-9.

36. Florindo AA, Hallal PC, Moura EC, Malta DC. Practice of physical activities and associated factors in adults, Brazil, 2006. Rev Saúde Pública 2009; 43 Suppl 2:65-73.

37. Florindo AA, Salvador EP, Reis RS, Guimarães VV. Perception of the environment and practice of physical activity by adults in a low socioeconomic area. Rev Saúde Pública 2011; 45:302-10.

38. Gobbi S, Caritá LP, Hirayama MS, Quadros Junior AC, Santos RF, Gobbi LTB. Behavior and barriers: physical activity in institutionalized elderly. Psicol Teor Pesqui 2008; 24:451-8.

39. Gomes GAO, Reis RS, Parra DC, Ribeiro I, Hino AAF Hallal PC, et al. Walking for leisure among adults from three Brazilian cities and its association with perceived environment attributes and personal factors. Int J Behav Nutr Phys Act 2011; 8:111

40. Guedes DP, Rocha GD, Silva AJRM, Carvalhal IM, Coelho EM. Effects of social and environmental determinants on overweight and obesity among Brazilian schoolchildren from a developing region. Rev Panam Salud Pública 2011; 30:295-302.

41. Hallal PC, Reis RS, Parra DC, Hoehner C, Brownson RC, Simões EJ. Association between perceived environmental attributes and physical activity among adults in Recife, Brazil. J Phys Act Health 2010; 7 Suppl 2:S213-22.

42. Hallal PC, Tenório MC, Tassitano RM, Reis RS, Carvalho YM, Cruz DK, et al. Evaluation of the Academia da Cidade program to promote physical activity in Recife, Pernambuco State, Brazil: perceptions of users and non-users. Cad Saúde Pública $2010 ; 26: 70-8$

43. Hino AAF, Reis RS, Ribeiro IC, Parra DC, Brownson RC, Fermino RC. Using observational methods to evaluate public open spaces and physical activity in Brazil. J Phys Act Health 2010; 7 Suppl 2: S146-54.

44. Hino AA, Reis RS, Sarmiento OL, Parra DC, Brownson RC. The built environment and recreational physical activity among adults in Curitiba, Brazil. Prev Med 2011; 52:419-22.

45. Malavasi LM, Duarte MFS, Both J, Reis RS. Escala de mobilidade ativa no ambiente comunitário news Brasil: retradução e reprodutibilidade. Rev Bras Cineantropom Desempenho Hum 2007; 9:339-50.
46. Martins MO, Petroski EL. Measurement of perceived barriers to physical activities: proposed research instrument. Rev Bras Cineantropom Desempenho Hum 2000; 2:58-65.

47. Matsudo V, Matsudo S, Andrade D, Araujo T, Andrade E, Oliveira LC, et al. Promotion of physical activity in a developing country: the Agita São Paulo experience. Public Health Nutr 2002; 5:253-61.

48. Matsudo SM, Matsudo VKR, Andrade DR, Araújo TL, Pratt M. Evaluation of a physical activity promotion program: the example of Agita São Paulo. Eval Program Plann 2006; 29:301-11.

49. Mendonça BC, Oliveira AC, Toscano JJO, Knuth AG, Borges TT, Malta DC, et al. Exposure to a community-wide physical activity promotion program and leisure-time physical activity in Aracaju, Brazil. J Phys Act Health 2010; 7 Suppl 2:S223-8.

50. Parra DC, McKenzie TL, Ribeiro IC, Ferreira Hino AA, Dreisinger M, Coniglio $\mathrm{K}$, et al. Assessing physical activity in public parks in Brazil using systematic observation. Am J Public Health 2010; 100:1420-6.

51. Petroski EL, Silva DAS, Reis RS, Pelegrini A. Stages of behavioral change and positive perception of the environment towards physical activity among urban park users. Motricidade 2009; 5:17-31.

52. Reis RS, Hino AA, Florindo AA, Añez CR, Domingues MR. Association between physical activity in parks and perceived environment: a study with adolescents. J Phys Act Health 2009; 6:503-9.

53. Reis RS, Hallal PC, Parra DC, Ribeiro IC, Brownson RC, Pratt M, et al. Promoting physical activity through community-wide policies and planning: findings from Curitiba, Brazil. J Phys Act Health 2010; 7 Suppl 2:S137-45.

54. Sallis JF, Bowles HR, Bauman A, Ainsworth BE, Bull FC, Craig CL, et al. Neighborhood environments and physical activity among adults in 11 countries. Am J Prev Med 2009; 36:484-90.

55. Salvador EP, Reis RS, Florindo AA. Practice of walking and its association with perceived environment among elderly Brazilians living in a region of low socioeconomic level. Int J Behav Nutr Phys Act 2010; 7:67.

56. Santos MS, Fermino RC, Reis RS, Cassou AC, Rodriguez-Añez CR. Barriers related to physical activity practice in adolescents. A focus-group study. Rev Bras Cineantropom Desempenho Hum 2010; 12:137-43.

57. Santos MS, Hino AA, Reis RS, Rodriguez-Añez CR. Prevalence of barriers for physical activity in adolescents. Rev Bras Epidemiol 2010; 13:94-104.

58. Scarinci IC, Silveira AF, Santos DF, Beech BM. Sociocultural factors associated with cigarette smoking among women in Brazilian worksites: a qualitative study. Health Promot Int 2007; 22:146-54.

59. Silva DAS, Petroski ED, Reis RS. Barriers to and facilitators of physical activity among public park visitors. Motriz Rev Educ Fís 2009; 15:219-27.

60. Silva KS, Vasques DG, Martins CO, Williams LA, Lopes AS. Active commuting: prevalence, barriers, and associated variables. J Phys Act Health 2011; 8:750-7. 
61. Silva SG, Silva MC, Nahas MV, Viana SL. Variables associated with leisure-time physical inactivity and main barriers to exercise among industrial workers in Southern Brazil. Cad Saúde Pública 2011; 27:249-59.

62. Simões EJ, Hallal P, Pratt M, Ramos L, Munk M, Damascena W, et al. Effects of a community-based, professionally supervised intervention on physical activity levels among residents of Recife, Brazil. Am J Public Health 2009; 99:68-75.

63. Gauthier E. Bibliometric analysis of scientific and technological research: a user's guide to the methodology. Ottawa: Statistics Canada; 1998.

64. van Lenthe FJ, De Bourdeaudhuij I, Klepp KI, Lien N, Moore L, Faggiano F, et al. Preventing socioeconomic inequalities in health behaviour in adolescents in Europe: background, design and methods of project TEENAGE. BMC Public Health 2009; 9:125.

65. Craig CL, Marshall AL, Sjöström M, Bauman AE, Booth ML, Ainsworth BE, et al. International physical activity questionnaire: 12-country reliability and validity. Med Sci Sports Exerc 2003; 35: 1381-95.

66. Saelens BE, Sallis JF, Black JB, Chen D. Neighborhood-based differences in physical activity: an environment scale evaluation. Am J Public Health 2003; 93:1552-8.

67. Alexander A, Bergman P, Hagstromer M, Sjostron M. IPAQ environmental module: reliability testing. J Public Health 2006; 14:76-80.

68. Cerin E, Saelens BE, Sallis JF, Frank LD. Neighborhood environment walkability scale: validity and development of a short form. Med Sci Sports Exerc 2006; 38:1682-91.

69. Caspi CE, Sorensen G, Subramanian SV, Kawachi I. The local food environment and diet: a systematic review. Health Place 2012; 18:1172-87.

70. Raine K, Spence JC, Church J, Boulé N, Slater L, Marko J, et al. State of the evidence review on urban health and healthy weights. Ottawa: Canadian Institute for Health Information; 2008.
71. Kuntsche E, Kuendig H, Gmel G. Alcohol outlet density, perceived availability and adolescent alcohol use: a multilevel structural equation model. J Epidemiol Community Health 2008; 62:811-6.

72. Ball K, Timperio AF, Crawford DA. Understanding environmental influences on nutrition and physical activity behaviors: where should we look and what should we count? Int J Behav Nutr Phys Act 2006; 3:33.

73. Ding D, Sallis JF, Kerr J, Lee S, Rosenberg DE. Neighborhood environment and physical activity among youth: a review. Am J Prev Med 2011; 41:442-55.

74. Diez-Roux AV. Investigating neighborhood and area effects on health. Am J Public Health 2001; 91:1783-9.

75. Montemurro GR, Berry TR, Spence JC, Nykiforuk C, Blanchard C, Cutumisu N. "Walkable by willpower": resident perceptions of neighbourhood environments. Health Place 2011; 17:895-901.

76. Brownson RC, Hoehner CM, Day K, Forsyth A, Sallis JF. Measuring the built environment for physical activity: state of the science. Am J Prev Med 2009; 36(4 Suppl):S99-123.

77. Florindo AA, Guimarães VV, Farias Júnior JC, Salvador EP, Sá TH, Reis RS, et al. Validation of the scale for evaluation of environment perception for physical activity practice in adults living in region of low socioeconomic level. Rev Bras Cineantropom Desempenho Hum 2012; 14:647-59.

78. Bengoechea GE, Spence JC, McGannon KR. Gender differences in perceived environmental correlates of physical activity. Int J Behav Nutr Phys Act 2005; 2:12.

79. McCormack GR, Shiell A. In search of causality: a systematic review of the relationship between the built environment and physical activity among adults. Inter J Behav Nutr Phys Act 2011; 8:125.

80. Foster S, Giles-Corti B. The built environment, neighborhood crime and constrained physical activity: an exploration of inconsistent findings. Prev Med 2008; 47:241-51.

Submitted on $01 /$ Mar/2013

Final version resubmitted on 12/Jun/2013

Approved on 24/Jun/2013 